\title{
COVID-19 y las desigualdades territoriales al interior de Áreas Metropolitanas de Valparaíso, Santiago y Concepción, Chile
}

\section{COVID-19 and territorial inequalities within the Metropolitan Areas of Valparaíso, Santiago and Concepción, Chile}

\section{COVID-19 e as desigualdades territoriais no interior das Áreas Metropolitanas de Valparaíso, Santiago e Concepción, Chile}

\author{
Manuel Fuenzalida \\ Universidad Alberto Hurtado, Departamento de Geografía, Chile \\ mfuenzal@uahurtadado.cl \\ https://orcid.org/0000-0003-3207-5763
}

\begin{abstract}
RESUMEN
Esta investigación analiza las desigualdades territoriales en morbilidad y mortalidad por COVID-19 al interior de las Áreas Metropolitanas de Valparaíso, Santiago y Concepción. Los datos se extraen del 40 informe epidemiológico de COVID-19 y para el proxy de los Determinantes Sociales de la Salud (DSS) se utilizan 8 variables o indicadores sociales de fuentes de información vigente del Ministerio de Desarrollo Social y el Instituto Nacional de Estadísticas. La metodología utilizada contempla AEDE uni y bi variado, la construcción de un Índice de DSS (IDSS) y la valoración de las relaciones de morbi-mortalidad por COVID-19 con el IDSS. El análisis de distribución espacial se valorará a partir de la posición de cada territorio en el cuadrante respectivo. Los resultados obtenidos permiten dilucidar brechas observables y comparables al interior de las comunas que componen las Áreas Metropolitanas investigadas. COVID-19, tiende a generar un mayor impacto en morbilidad cuando empeoran condiciones de pobreza multidimensional y hacinamiento. En términos de mortalidad, la distribución territorial de grupos de riesgos condiciona el impacto y la posible definición de estrategias diferenciadas para limitar el daño en salud.
\end{abstract}

\begin{abstract}
This research analyzes the territorial inequalities in morbidity and mortality due to COVID-19 within the Metropolitan Areas of Valparaíso, Santiago and Concepción. The data is extracted from the 40th epidemiological report of COVID-19 and for the proxy of the Social Determinants of Health (DSS) 8 variables or social indicators are used from current information sources of the Ministry of Social Development and the National Institute of Statistics. The methodology used contemplates Exploratory Analysis of Spatial Data uni and bi varied, the construction of a DSS Index (IDSS) and the assessment of the morbidity-mortality relationships due to COVID-19 with the IDSS. The analysis of spatial distribution will be assessed from the position of each territory in the respective quadrant. The results obtained allow us to elucidate observable and comparable gaps within the communes that make up the Metropolitan Areas investigated. COVID-19 tends to generate a greater impact

1 Trabajo realizado en el marco del Proyecto ANID0476.
\end{abstract}

Recibido:31/08/2020 - Aceptado: 02/10/2020 - Publicado: 22/02/2021

\section{Citar como:}

Fuenzalida, M. (2020). COVID-19 y las desigualdades territoriales al interior de Áreas Metropolitanas de Valparaíso, Santiago y Concepción, Chile. Espiral, revista de geografías y ciencias sociales, 2(4), 079 - 089. http://dx.doi.org/10.15381/espiral. v2i4.19535

C Los autores. Este artículo es publicado por Espiral, revista de geografías y ciencias sociales de la Universidad Nacional Mayor de San Marcos. Este es un artículo de acceso abierto, distribuido bajo los términos de la licencia Creative Commons Atribucion- No Comercia_Compartir Igual 4.0 Internacional. (http://creativecommons.org/licenses/by-nc-sa/4.0/) que permite el uso no comercial, distribución y reproducción en cualquier medio, siempre que la obra original sea debidamente citada. 
on morbidity when conditions of multidimensional poverty and overcrowding worsen. In terms of mortality, the territorial distribution of risk groups determines the impact and the possible definition of differentiated strategies to limit health damage.

\title{
RESUMO
}

\begin{abstract}
A pesquisa analisa as desigualdades territoriais em morbilidade e mortalidade pela COVID-19 no interior das Áreas Metropolitanas de Valparaíso, Santiago e Concepción. Os dados se extraem do 40으 informe epidemiológico da COVID-19 e pelo proxy dos Determinantes Socias da Saúde (DSS) se utilizam 8 variáveis ou indicadores sociais de fontes de informação vigente do Ministério de Desenvolvimento Social e o Instituto Nacional de Estatísticas. A metodologia utilizada contempla Análises Exploratórios de Dados Espaciais uni e bi variado, a construção de um Índice de DSS (IDSS) e a valoração das relações de morbi-mortalidade pela COVID-19 com IDSS. A análises de distribuição espacial se valorará a partir da posição de cada território no quadrante respetivo. Os resultados obtidos permitem dilucidar brechas observáveis e comparáveis ao interior das comunas que compõem as Áreas Metropolitanas pesquisadas. A COVID-19, tende a gerar um maior impacto em morbilidade quando empioram condições de pobreza multidimensional e superlotação. Em termos de mortalidade, a distribuição territorial de grupos de riscos condiciona o impacto e a possível definição de estratégias diferenciadas para limitar o dano em saúde.
\end{abstract}

PALABRAS CLAVE: Desigualdad territorial; COVID-19; Chile.

KEYWORDS: Territorial inequality; COVID-19; Chile.

PALAVRAS-CHAVE: Desigualdade territorial; COVID-19; Chile.

\section{Introducción}

El 11 de marzo de 2020, la Organización Mundial de la Salud (OMS) caracteriza el COVID-19 como una pandemia, tras reportar más de 100 mil casos en 114 países. En Chile, la evolución de la morbilidad indica que el día 2 de marzo se registró el primer caso. El 20 de abril se superaron los 10 mil casos. El 2 de junio se superaron los $100 \mathrm{mil}$ casos. El 25 de octubre se superaron los 500 mil casos. En relación a la evolución de la mortalidad, la primera muerte en el país aconteció el 23 de marzo. El día 17 de abril se contabilizaban más de 100 muertos. El 1 de junio las muertes superan las mil. El 10 de agosto, superaron las $10 \mathrm{mil}$. El 21 de noviembre superaron las $15 \mathrm{mil}$.

Geografía y salud es un binomio que, en los últimos 30 años, ha reunido suficiente evidencia para comprender que los efectos en salud varían geográficamente $y$, en particular, que los residentes en territorios más vulnerables o desfavorecidos tienen peor salud que aquellos que habitan zonas menos vulnerables; cuestión que se está acentuando en muchos países (Smith y Easterlow, 2005; Pearce y Kingham, 2008; Richardson et al., 2013).

En términos empíricos de desigualdad, América Latina ostenta los peores valores y las mayores diferencias entre grupos aventajados versus grupos en desventaja. Por ejemplo, en nuestro país el ingreso per cápita del 1\% más rico es 40 veces mayor que el ingreso per cápita del $81 \%$ de la población (López et al., 2013). Una prueba de la magnitud de las desigualdades en salud en las áreas urbanas en la región es posible encontrarla en el proyecto Salud Urbana en América Latina (SALURBAL), que estudió seis grandes ciudades de América Latina (Buenos Aires, Belo Horizonte, Santiago, San José, Ciudad de México y Ciudad de Panamá) y encontró grandes diferencias espaciales en la esperanza de vida al nacer dependiendo del nivel socioeconómico del lugar de residencia (Bilal et al., 2019).

Así, el propósito del presente trabajo tiene como objetivo desarrollar un diagnóstico cuantitativo de las desigualdades existentes en morbi y mortalidad asociada a COVID-19 al interior de las tres principales Áreas Metropolitanas del país, siendo estas las de Valparaíso (AMV), Santiago (AMS) y Concepción (AMC). La distribución territorial de la desigualdad será valorada a partir de intervalos de desviación 
estándar, prestando una especial atención a los territorios que quedan en los intervalos extremos (Fuenzalida, 2012).

En términos teóricos y conceptuales, la Geografía se ha preocupado por las desigualdades territoriales y los factores que la definen en términos culturales, demográficos, sociales y económicos, además de los propios condicionantes naturales (Barcellos y Buzai, 2006; Santana et al., 2016). Una de las vías de análisis corresponde al uso de Sistemas de Información Geográfica y la estadística espacial, en donde las unidades de observación adquieren un valor y estos son caracterizados a partir de su posición en una distribución segmentada de un quintil o decil determinado.

La construcción argumental por parte de la OMS del marco conceptual de las determinantes sociales de la salud (DSS), ha permitido valorar y evaluar como los contextos en que las personas nacen, crecen, viven, trabajan y envejecen, más las capacidades instaladas del sistema sanitario, influencian o impactan en el resultado de salud de las personas.

Las DSS se dividen en dos grupos. Primero, están aquellas que hacen referencia a las de tipo estructural (DSSE) que refieren a los ejes guías de las circunstancias de vida de las personas, como por ejemplo las políticas sociales (vivienda, trabajo), las políticas públicas (salud, educación), o los valores culturales de la población. Aquí están presentes todo lo concerniente al ámbito sociopolítico y económico del Estado. Segundo, están aquellas que hacen referencia a las de tipo intermedias (DSSI) que corresponden a aquellas variables de carácter individual como pueden ser las circunstancias materiales de las personas (condiciones de vida y trabajo), los factores biológicos (sexo, edad) y los factores psicosociales (problemas de relación, estrés), resultando de lo anterior, en impactos directos en desigualdades en salud y en bienestar (Fuenzalida et al., 2013).

De esta forma, el estudio sistemático de las DSS, ha permitido evidenciar que las desigualdades socioeconómicas contribuyen a una disparidad en la carga de enfermedades entre las poblaciones desfavorecidas y favorecidas, patrón que se repite en países de ingresos bajos, medianos y altos. Así lo deja de manifiesto el estudio de Sommer et al. (2015), que realizó una revisión sistemática para evaluar de forma objetiva la evidencia disponible sobre las desigualdades socioeconómicas en relación con la morbilidad y mortalidad de enfermedades y sus factores de riesgo.

Como bien describe Patel et al. (2020), las personas de nivel socioeconómico bajo (NSB) presentan una serie de factores que aumentan su exposición al COVID-19. En primer lugar, las personas económicamente desfavorecidas tienen más probabilidades de vivir en viviendas con hacinamiento, en donde existen más dificultades para el cumplimiento del distanciamiento social. En segundo lugar, las personas de NSB a menudo están empleadas en ocupaciones que no brindan oportunidades para teletrabajar. En tercer lugar, los que pertenecen a grupos de NSB tienen más probabilidades de tener condiciones de trabajo e ingresos inestables, condiciones exacerbadas por las respuestas al COVID-19 y sus consecuencias. En cuarto lugar, las personas de NSB se presentan a los servicios de salud en una etapa más avanzada de la enfermedad, lo que da como resultado peores resultados de salud. Finalmente, existe evidencia emergente de que la hipertensión y la diabetes son factores de riesgo de muerte por COVID-19 (Guam et al., 2020).

Al día de hoy, el avance en la captura, producción y manipulación de información territorial con fines de análisis social, permite observar empíricamente como grupos sociales residentes en determinados territorios, nacen, crecen, viven, trabajan y envejecen dentro de límites administrativos establecidos. A partir de la existencia de tres o más unidades territoriales de análisis, es posible comprender y asociar desigualdades en salud, así como todos los datos del contexto social y la distribución de la asistencia sanitaria (Charreire y Combier, 2009). 
De esta forma, las desigualdades en salud entre grupos de distinto NSB, sean estos medios por educación, ocupación o ingresos, constituye uno de los principales desafíos para la salud pública. Por ejemplo, en el estudio de Mackenbach et al. (2008), entre veintidós países de europeos, las tasas de muerte y las peores autoevaluaciones de la salud fueron sustancialmente más altas en los grupos de nivel socioeconómico más bajo, pero la magnitud de las desigualdades entre los grupos de nivel socioeconómico más alto y más bajo fue mucho mayor en algunos países que en otros. Para América Latina y El Caribe, el estudio de Vincens et al. (2018), establece que existe un claro gradiente social en salud, independiente de la heterogeneidad a nivel de país y del capital social a nivel individual.

Por lo antecedentes anteriormente expuestos, es válido preguntarse si COVID-19 está impactando a todos por igual o, por el contrario, existen grupos que están exhibiendo peores resultados y presentando una mayor carga en morbilidad y mortalidad.

\section{Metodología}

\section{1. Área de estudio}

El área de estudio corresponde a las tres principales Áreas Metropolitanas del Chile, las que son el Áreas Metropolitana de Valparaíso (AMV), Santiago (AMS) y Concepción (AMC). Su distribución territorial se puede observar en la figura 1. El AMV está formada por 5 unidades sub-nacionales correspondientes a comunas, albergando en su conjunto 1.029.387 residentes. El AMS está formada por 34 comunas que concentran 6.976.740 residentes. El AMC está formada por 7 comunas que suman 780.661 residentes.
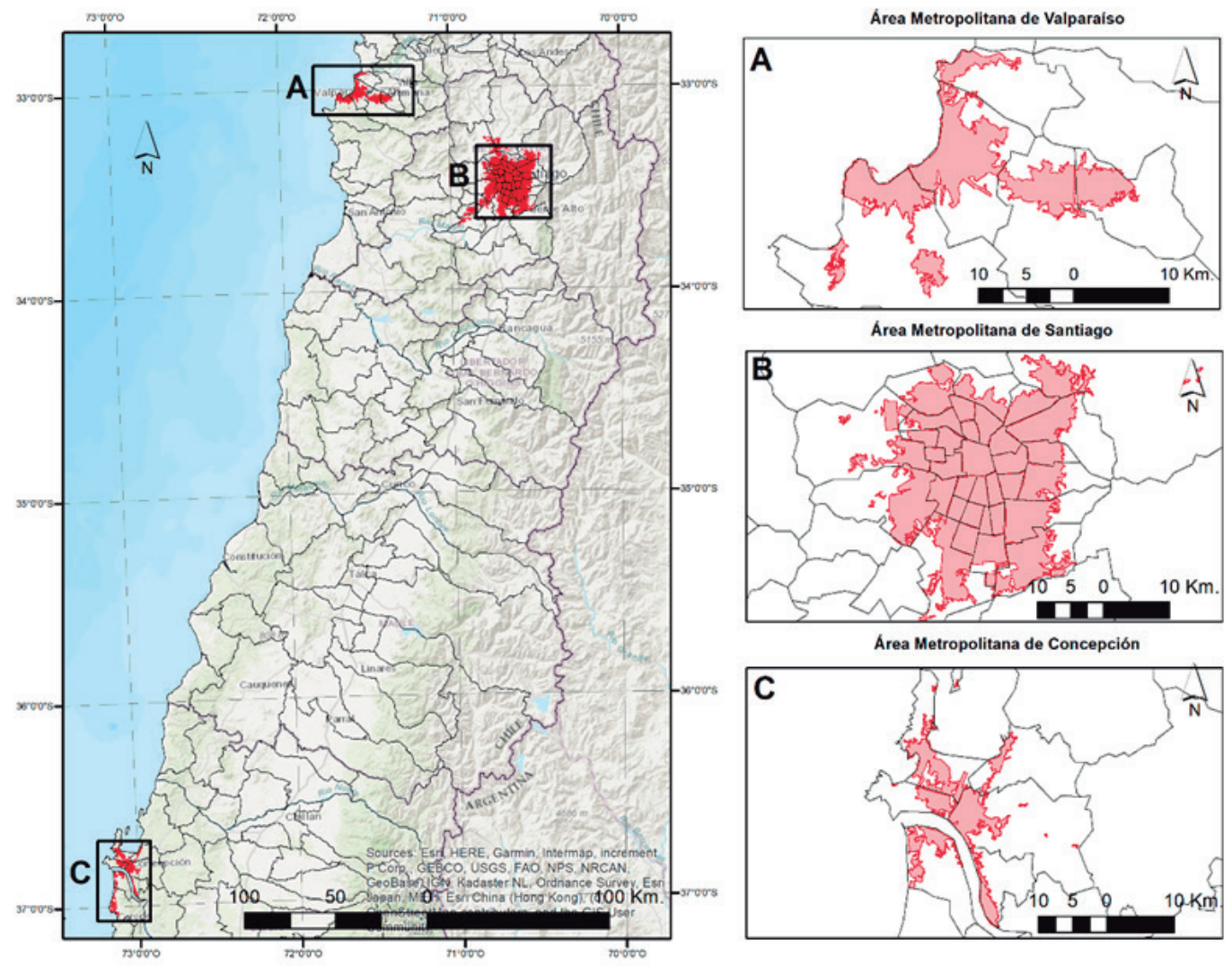

Figura 1. Localización geográfica del área de estudio

Fuente: Proceso propio, 2020 en el software ArcMap®. 


\subsection{Selección de variables}

Los datos de morbilidad y mortalidad son extraídos del $40^{\circ}$ informe epidemiológico de COVID-19 (07/08/2020), Departamento de Epidemiología, Ministerio de Salud de Chile. La elección de este informe en particular se debe a que recoge el comportamiento de la pandemia durante la "primera ola". El dato de morbilidad corresponde a las tasas de incidencia (TMorb) de personas con COVID-19 por 100.000 habitantes, considerando comuna de residencia y utilizando la actualización de proyecciones de población del Instituto Nacional de Estadísticas (INE) 2020, en base al Censo 2017. El dato de mortalidad (TMort) corresponde a la proporción de personas con COVID-19 que fallecen con respecto al total de la población. Se expresa amplificada a 100.000 habitantes.

Para las variables e indicadores sociales de componen el proxy de los Determinantes Sociales de Salud se utilizan las descritas en la tabla 1.

Tabla 1. Variables e indicadores sociales de Determinantes Sociales de Salud

\begin{tabular}{lllll}
\hline \multicolumn{1}{c}{ Indicador } & $\mathbf{n}$ & \multicolumn{1}{c}{ Nombre } & V. Max & Abreviatura \\
\hline & 1 & Porcentaje de Pobreza Multidimensional & Costo & PMULTI \\
Determinantes & 2 & Porcentaje Carente de Servicios Básicos & Costo & CSB \\
Estructurales & 3 & Establecimientos hospitalarios & Beneficio & HOSP \\
& 4 & Establecimientos Atención Primaria Salud & Beneficio & APS \\
Determinantes & 1 & Porcentaje de hogares Tramo 40 & Costo & PHT40 \\
Intermedias & 2 & Porcentaje de hogares con Hacinamiento & Costo & PHH \\
& 3 & Porcentaje de población 60 a 79 años & Costo & P60a79 \\
\hline
\end{tabular}

Fuente: Elaboración propia con base en Fuenzalida (2018).

- Porcentaje de Pobreza Multidimensional (PMULTI): Identifica múltiples carencias a nivel de los hogares y las personas en los ámbitos de la salud, la educación y el nivel de vida. Cada miembro de una familia es clasificado como pobre o no pobre en función del número de carencias que experimente su hogar. Estos datos se agregan a nivel comunal para conformar el indicador de la pobreza multidimensional. Fuente: Encuesta de Caracterización Socioeconómica Nacional (CASEN) 2017.

- Población Carente de Servicios Básicos (CSB): Identifica el porcentaje de personas carentes de servicios básicos. Fuente: Registro Social de Hogares (RSH) marzo de 2020.

- Establecimientos hospitalarios (HOSP): Identifica el número de establecimientos hospitalarios disponibles en cada comuna del país y que pertenecen al Sistema Nacional de Servicios de Salud (SNSS). Fuente: Ministerio de Salud 2020.

- Establecimientos Atención Primara Salud (APS): Identifica el número de establecimientos de atención primaria de salud disponibles en cada comuna del país y que representa el primer nivel de contacto de los individuos, la familia y la comunidad con el sistema público. Fuente: Ministerio de Salud 2020.

- Porcentaje de hogares Tramo 40 (PHT40): Identifica el porcentaje de hogares a nivel comunal que están clasificados dentro del $40 \%$ de hogares de menores ingresos o mayor vulnerabilidad socioeconómica en el país. Fuente: Registro Social de Hogares (RSH) marzo de 2020.

- Porcentaje de hogares con Hacinamiento (PHH): Identifica el porcentaje de hogares hacinados a nivel comunal. Fuente: Registro Social de Hogares (RSH) marzo de 2020. 
- Porcentaje de población 60 a 79 años (P60a79): Identifica el porcentaje de población entre 60 y 79 años de edad a nivel comunal. Fuente: Censo de Población y Vivienda 2017.

- Porcentaje de población 80 años y más (P80ym): Identifica el porcentaje de población de 80 y más años de edad a nivel comunal. Fuente: Censo de Población y Vivienda 2017.

\subsection{Análisis de datos}

Para detectar brechas territoriales en los DSS y su relación con la Morbilidad y Mortalidad por COVID-19, se utilizará un protocolo metodológico que consta de tres pasos concatenados. Estos serán implementados con free software GeoDA.

El Paso 1, corresponde al uso del Análisis Exploratorio de Datos Espaciales (AEDE) para conocer el comportamiento de las variables de morbilidad y mortalidad en relación a los DSS. En AEDE el mapa está integrado en el esquema general de análisis, junto con el resto de gráficos dinámicos. Se utilizará el diagrama de caja y la matriz de diagramas de dispersión para describir el comportamiento por separado de morbilidad y mortalidad con las variables DSS estructurales (DSSE) y DSS intermedias (DSSI).

En el paso 2, se estandarizarán las variables e indicadores sociales de Determinantes Sociales de Salud siguiendo la fórmula propuesta en Fuenzalida y Cobs (2013) e interpretando los valores cercanos cero (0) como deseado y los valores cercanos a 1 como indeseado. Se procederá a realizar una sumatoria de las variables estandarizadas de DSSE y DSSI, resultando un Índice DSS (IDSS) con un intervalo teórico entre cero (0) -extrema ventaja- y ocho (8) -extrema desventaja-.

En el paso 3, se procederá a construir un diagrama de dispersión que en el eje de las abscisas $(x)$ posicionará los valores del IDSS y en el eje de las ordenadas (y) posicionará los datos de morbilidad (TMorb) y mortalidad (TMort) por COVID-19. A partir de la media de cada variable, se construirán cuadrantes de valorización, que seguirá la interpretación de la figura 2.

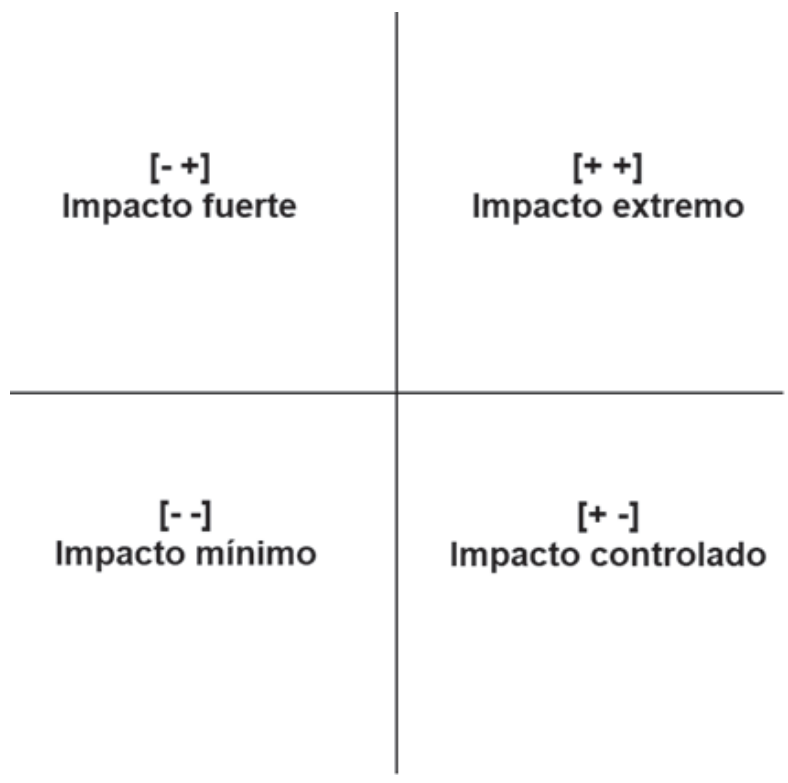

Figura 2. Valoración de las relaciones morbi-mortalidad por COVID-19 y los Determinantes Sociales de Salud

Fuente: Elaboración propia. 


\section{Resultados}

El resultado del análisis univariado de cada variable de interés se puede observar en la tabla 2. El estadístico intercuartil (IQR) indica que en el 50\% central de las comunas que componen las áreas metropolitanas, la variación entre comunas para la Tmorb varía como máximo 2.974 casos por 100.000 habitantes. Para la TMort varía como máximo 99,9 casos por 100.000 habitantes. El resultado del mapa diagrama de caja para morbilidad y mortalidad por COVID-19 se observa en la figura 3. De las tres áreas metropolitanas analizadas, la de Santiago es la que concentra el 50\% de los valores superiores tanto para morbilidad como mortalidad, considerando que el patrón territorial no es idéntico.

Tabla 2. Resumen de estadísticas para variables

\begin{tabular}{lcccccccccc}
\hline & TMorb & TMort & PMULTI & CSB & HOSP & APS & PHT40 & PHH & P60a79 & P80ym \\
\hline Min & 704,6 & 5,6 & 3,4 & 0,5 & 0 & 1 & 25,9 & 2 & 8,1 & 0,9 \\
Max & $5.673,3$ & 186,5 & 37,5 & 20,1 & 6 & 16 & 59,7 & 20,9 & 18 & 5,3 \\
Q1 & $1.401,2$ & 30,4 & 13,5 & 5,5 & 0 & 4 & 49,9 & 11,4 & 12,6 & 2 \\
Median & $3.323,9$ & 93,8 & 18,6 & 7,8 & 0 & 5,5 & 51,9 & 14,3 & 14,4 & 3 \\
Q3 & $4.375,2$ & 130,3 & 24,5 & 9,6 & 1 & 8 & 55,2 & 15,8 & 15,3 & 3,8 \\
IQR & 2.974 & 99,9 & 11 & 4,1 & 1 & 4 & 5,3 & 4,4 & 2,7 & 1,8 \\
Mean & 2983,4 & 90,4 & 19,1 & 7,8 & 0,8 & 6,2 & 51 & 13,5 & 13,9 & 2,9 \\
s.d. & $1.492,2$ & 56,9 & 8,0 & 4,2 & 1,3 & 3,6 & 7,2 & 3,8 & 2,1 & 1,1 \\
\hline
\end{tabular}

Fuente: Proceso propio, 2020 en el software GeoDa®.

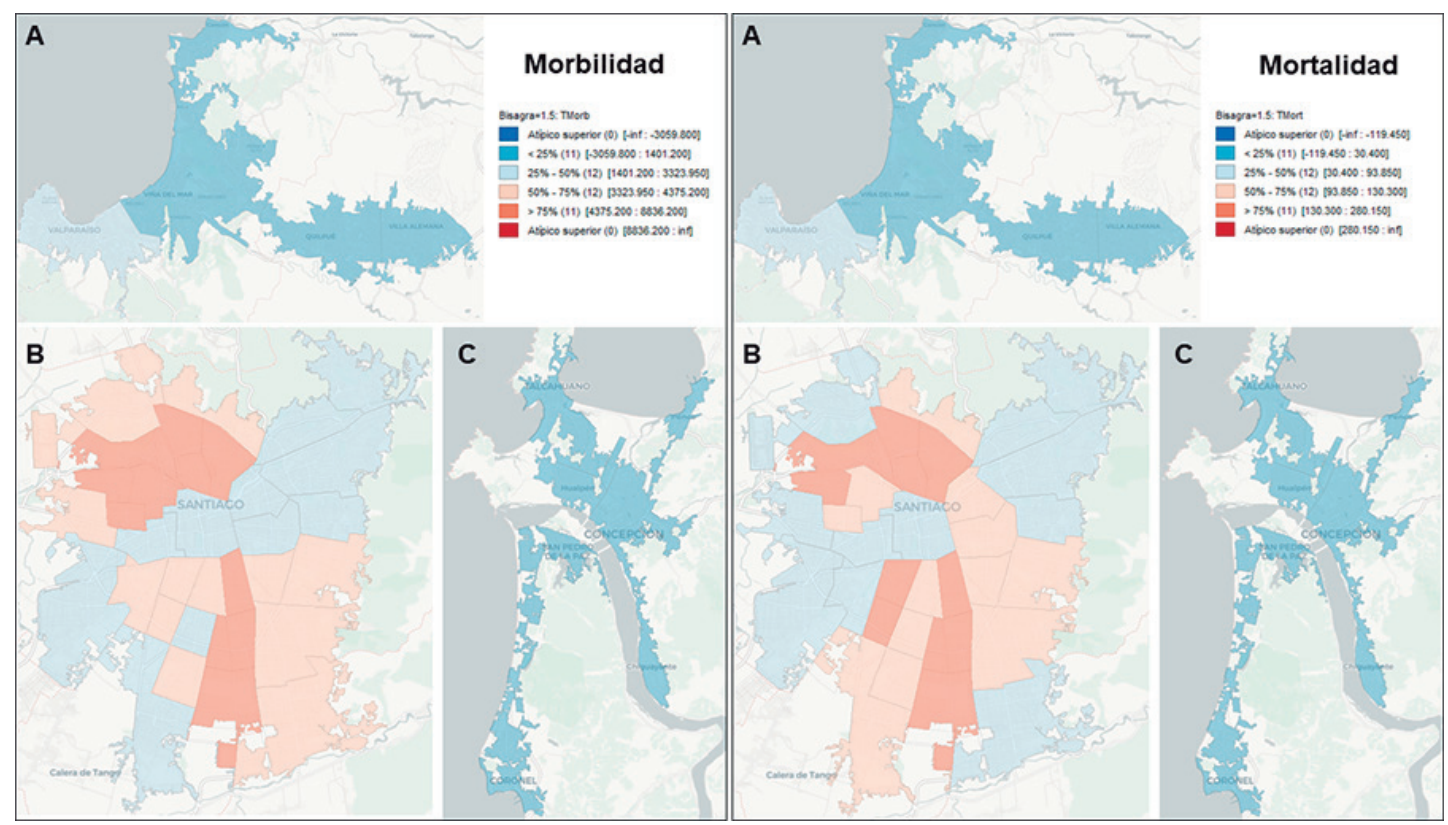

Figura 3. Mapa diagrama de caja para morbilidad y mortalidad por COVID-19

Fuente: Proceso propio, 2020 en el software GeoDa®. Nota: A: Área Metropolitana de Valparaíso. B: Área Metropolitana de Santiago. C: Área Metropolita de Concepción.

El resultado del análisis bivariado para morbilidad y mortalidad por COVID-19 y las DDS estructurales se puede observar en la figura 4. A nivel territorial, mientras mayor sea la tasa de morbilidad se espera una mayor tasa de mortalidad. Eso se acentúa con valores más altos de pobreza multidimensional y carencia de servicios básicos. Morbilidad y mortalidad tienden a ser menores a medida de una mayor existencia de infraestructura hospitalaria y de atención primaria de salud. 


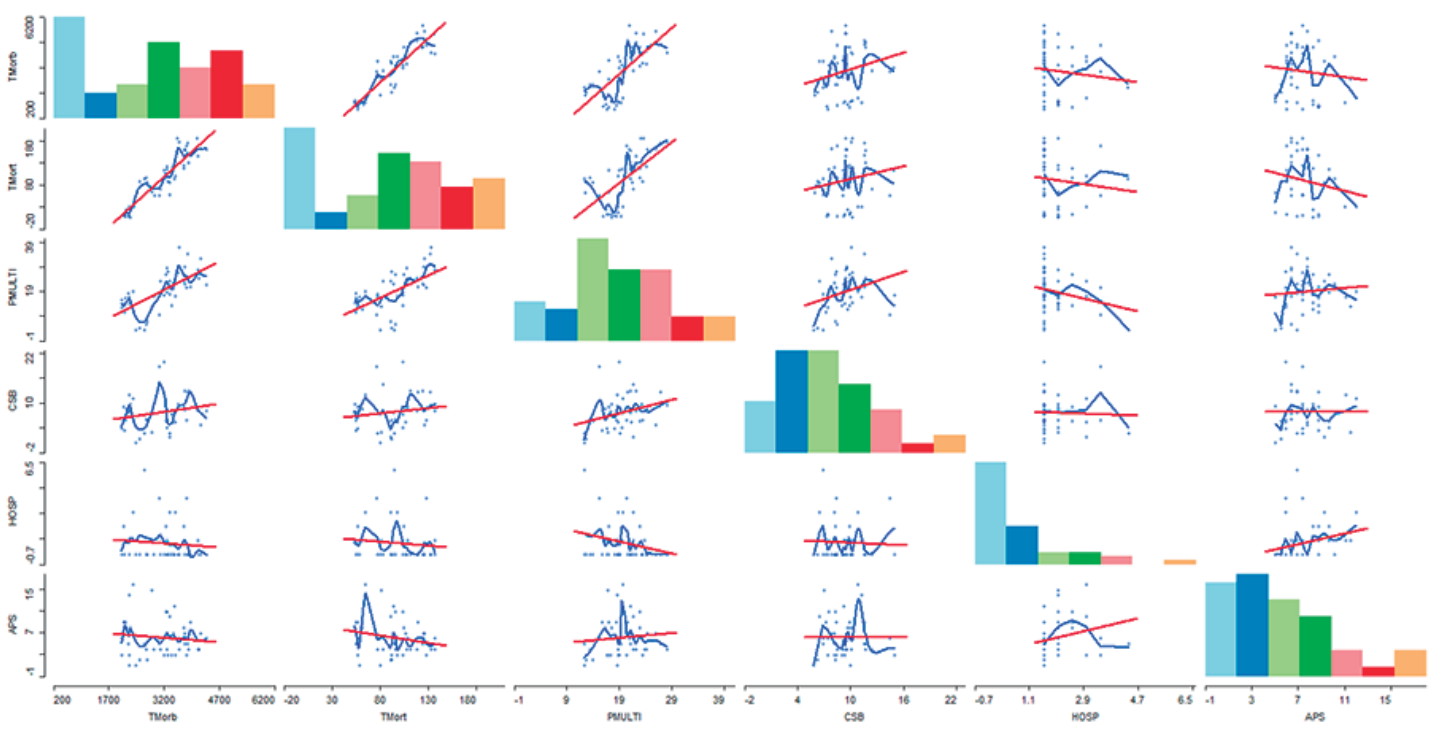

Figura 4. Diagramas de dispersión Morbilidad y Mortalidad por COVID-19 versus variables DSS estructurales

Fuente: Proceso propio, 2020 en el software GeoDa.

Nota: Cada punto es una comuna. Línea roja: Alisado lineal. Línea azul: Alisado Lowess.

El resultado del análisis bivariado para morbilidad y mortalidad por COVID-19 y las DDS intermedias se puede observar en la figura 5. A nivel territorial, mientras mayor sea la tasa de morbilidad se espera una mayor existencia de hogares pertenecientes a los primeros 4 deciles de ingreso y mayores hogares con hacinamiento. La morbilidad general tiende a disminuir en presencia de población de riesgo sobre 60 años. En relación a la tasa de mortalidad, tiende a presentar peores resultados en territorios que concentran los 4 primeros deciles de ingreso, hogares con hacinamiento y mayor proporción de población de riesgo sobre 60 años.

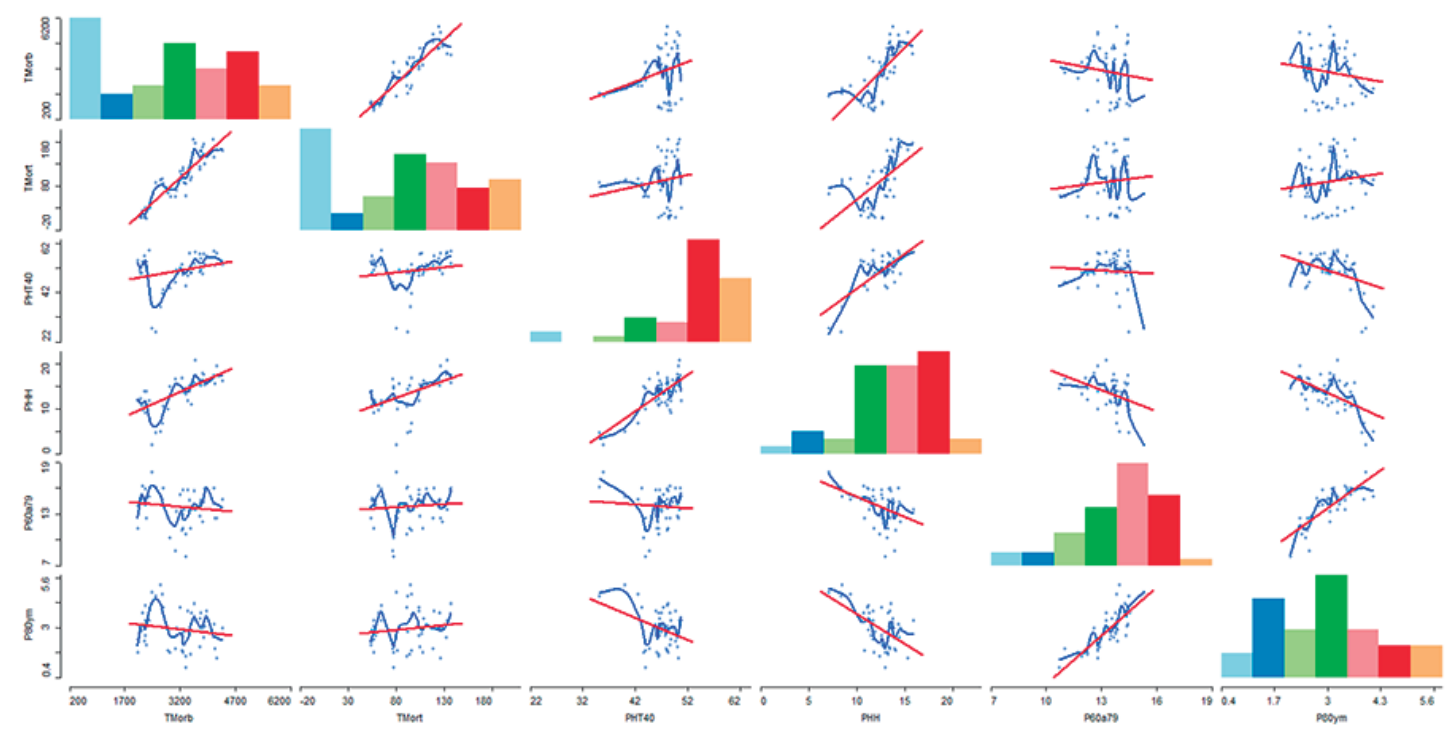

Figura 5. Diagramas de dispersión Morbilidad y Mortalidad por COVID-19 versus variables DSS intermedias

Fuente: Proceso propio, 2020 en el software GeoDa.

Nota: Cada punto es una comuna. Línea roja: Alisado lineal. Línea azul: Alisado Lowess.

La sumatoria de las variables estandarizadas de DDS estructurales y DSS intermedias permitieron la construcción del Índice DSS, que como valor mínimo ostenta 3 y 
máximo 6.5. En las medidas de centralidad encontramos la mediana 4,8 y el promedio 4,78. Como medida de dispersión tenemos la desviación estándar 0,8. La posición de cada territorio en el diagrama de dispersión se puede observar en la figura 6. Para el caso de la morbilidad por COVID-19, 19 territorios se posicionan en el cuadrante $[++]$ cuya valorización del impacto de los DSS sobre la incidencia de la enfermedad es considerado un impacto extremo. 6 territorios se posicionan en el cuadrante [+-] valorado como un impacto controlado de los DSS sobre la incidencia de la enfermedad. 14 territorios se posicionan en el cuadrante [--] valorado como un impacto mínimo de los DSS sobre la incidencia de la enfermedad. Finalmente, 7 territorios se posicionan en el cuadrante $[-+]$ valorado como un impacto fuerte de los DSS sobre la incidencia de la enfermedad.

Para el caso de la mortalidad por COVID-19, 17 territorios se posicionan en el cuadrante $[++] .8$ territorios se posicionan en el cuadrante [+-]. 14 territorios se posicionan en el cuadrante [--]. 7 territorios se posicionan en el cuadrante [-+].
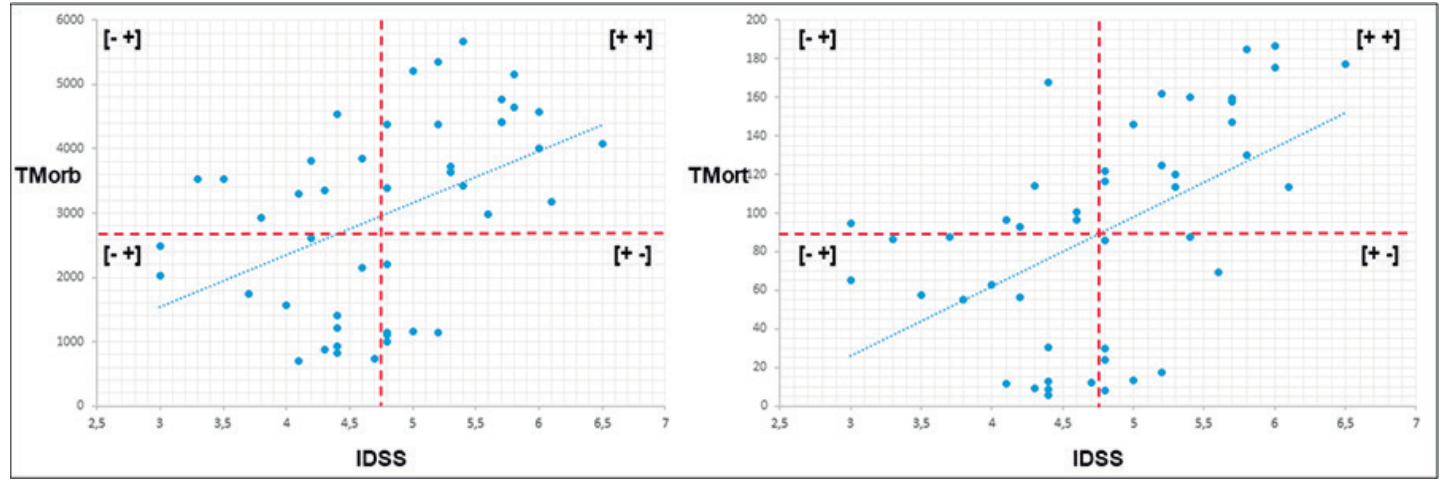

Figura 6. Valoración de las relaciones morbilidad y mortalidad por COVID-19 y los Determinantes Sociales de Salud

Fuente: Proceso propio, 2020 en el software GeoDa®.

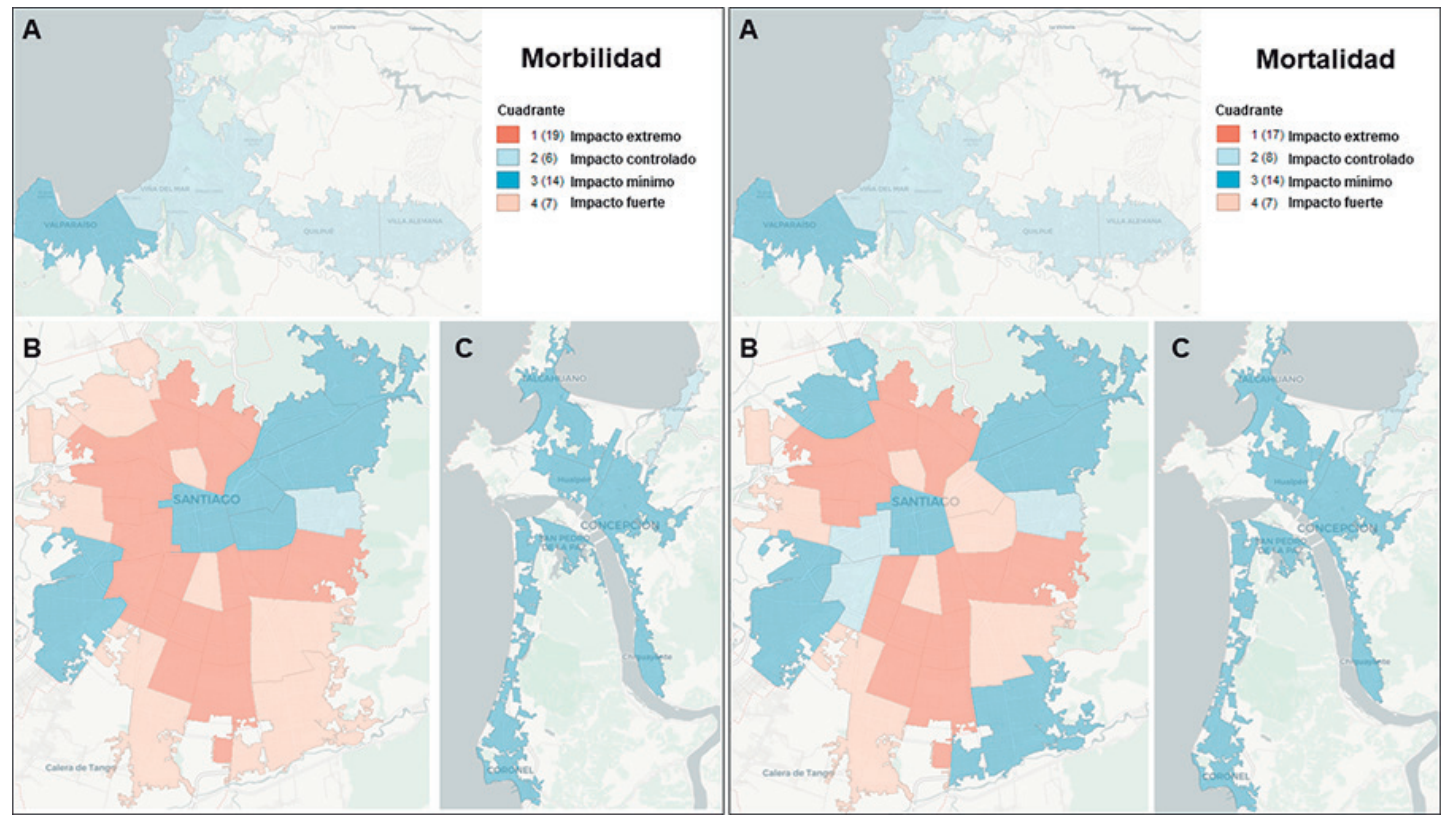

Figura 7. Mapa de valoración de las relaciones morbi-mortalidad por COVID-19 y los Determinantes Sociales de la Salud

Fuente: Proceso propio, 2020 en el software GeoDa®. Nota: A: Área Metropolitana de Valparaíso. B: Área Metropolitana de Santiago. C: Área Metropolita de Concepción. 
El patrón territorial de la valoración de las relaciones morbi-mortalidad por COVID-19 y los Determinantes Sociales de la Salud se pueden observar en la figura 7. El Área Metropolitana de Valparaíso exhibe mayormente impactos controlado y mínimo, plausiblemente relacionados a una menor proporción de población y un mejor equilibrio en la infraestructura sanitaria de hospitales y APS. El Área Metropolitana de Concepción muestra mayoritariamente impactos mínimos, en línea con las razones del AMV. El Área Metropolitana de Santiago presenta un impacto extremo de los DSS sobre incidencia de la enfermedad y también en su mortalidad, siendo las comunas que presentan ambas condiciones las de Cerro Navia, Conchalí, El Bosque, Huechuraba, La Cisterna, La Granja, La Pintana, Lo Espejo, Lo Prado, Macul, Pedro Aguirre Cerda, Peñalolén, Quinta Normal, Recoleta, Renca, San Joaquín y San Ramón. El anterior listado territorial concentra los peores valores para los DSSE Porcentaje de Pobreza Multidimensional, Establecimientos hospitalarios, Establecimientos Atención Primaria Salud, y para los DSSI Porcentaje de hogares Tramo 40 y Porcentaje de hogares con Hacinamiento.

\section{Conclusiones}

El estatus socioeconómico es quizás el concepto central que aglutina el conjunto de determinantes sociales que dan forma a la salud (Stringhini et al., 2017). Por ejemplo, el estatus socioeconómico influye en el lugar de residencia, el cuál es peor para condiciones de vivienda, hacinamiento y saneamiento los cuales ponen a las personas en mayor riesgo de transmisión de COVID-19 (Rollston y Galea, 2020). El estatus socioeconómico también influye en la posibilidad de acceso a servicios sanitarios de APS u hospitalarios, lo que afecta a la detección y tratamiento oportuno de COVID-19 (Rozenfeld et al., 2020).

Los resultados obtenidos permiten dilucidar brechas observables y comparables al interior de las comunas que componen las Áreas Metropolitanas investigadas. COVID-19, tiende a generar un mayor impacto en morbilidad cuando empeoran condiciones de pobreza multidimensional y hacinamiento. En términos de mortalidad, la distribución territorial de grupos de riesgos condiciona el impacto y la posible definición de estrategias diferenciadas para limitar el daño en salud. La existencia de instalaciones y equipamiento sanitario tiende a limitar el impacto en morbilidad y mortalidad por COVID-19.

El protocolo metodológico desarrollado en tres pasos concatenados e implementado con free software GeoDA, permite de forma eficaz priorizar los territorios donde el impacto de los DSS en fuerte o extremo en relación con la incidencia de la enfermedad y su mortalidad.

Para el caso del Área Metropolitana de Santiago, son 17 comunas las que concentran los peores valores de morbilidad y mortalidad por COVID-19, las que se caracterizan por presentar desventaja en 5 de las 8 variables o indicadores analizados.

Es por ello que es posible concluir que COVID-19 no impacta a todos por igual. Aquellos territorios que presentan mayor vulnerabilidad están exhibiendo peores resultados en morbilidad y mortalidad.

\section{Referencias Bibliográficas}

Barcellos, C. y Buzai, G. (2006). La dimensión espacial de las desigualdades sociales en salud. Aspectos de su evolución conceptual y metodológica. Anuario de la División Geografía de la Universidad Nacional de Luján. Luján. pp. 275-292.

Bilal, U., Alazraqui, M., Caiaffa, W., Lopez-Olmedo, N., Martinez-Folgar, K., Miranda, J., Rodriguez, D., Vives, A. y Diez-Roux, A. (2019). Inequalities in life expectancy in six large 
Latin American cities from the SALURBAL study: an ecological analysis. Lancet Planet Health; 3: e503-10.

Charreire, H., y Combier, E. (2009). Poor prenatal care in an urban area: A geographic analysis. Health E Place, Vol. 15, pp 412 - pp 419.

Fuenzalida, M. (2012). Evaluación de desigualdades espaciales y cambios temporales en el desarrollo regional. En Moreno, A., Buzai, G. y Fuenzalida, M. (Eds): Sistemas de Información Geográfica. Aplicaciones en diagnósticos territoriales y decisiones geoambientales. Madrid, Editorial RA-MA.

Fuenzalida, M. (2018). Detección de brechas territoriales utilizando análisis exploratorio de datos espaciales: el caso de los determinantes sociales de la salud, Chile. En: Plata Rocha y Pineda Jaimes. Análisis territorial mediante Tecnologías de la Información Geográfica. Ciudad de México, pp.201-216.

Fuenzalida, M. y Cobs, V. (2013). La perspectiva del análisis espacial en la herramienta sig: una revisión desde la geografía hacia las ciencias sociales. Persona y Sociedad, 27 (3), 33-52.

Fuenzalida, M., Cobs, V., \& Guerrero, R. (2013). El territorio como unidad de análisis en estudios sobre las determinantes sociales de la salud. Argos, 30(59), 086-106.

Guan W., Liang W., Zhao Y., Liang H., Chen Z., Li Y. (2020). Comorbidity and its impact on 1590 patients with covid-19 in China: a nationwide analysis. Eur Respir J., Mar 26;55(5).

López, R., Figueroa, E. y Gutiérrez, P. (2013). La 'parte del león': nuevas estimaciones de la participación de los súper ricos en el ingreso de Chile, serie "Documentos de Trabajo", núm. 32, Departamento de Economía, Universidad de Chile.

Mackenbach, J. P., Stirbu, I., Roskam, A. J. R., Schaap, M. M., Menvielle, G., Leinsalu, M., \& Kunst, A. E. (2008). Socioeconomic inequalities in health in 22 European countries. New England journal of medicine, 358(23), 2468-2481.

Patel, J.A., Nielsen, F. y Badiani, A. et al. (2020). Poverty, inequality and COVID-19: the forgotten vulnerable. Public Health, 183: 110-111.

Pearce, J., y Kingham ,S. (2008). Environmental inequalities in New Zealand: A national study of air pollution and environmental justice. Geoforum, 39:980 - 993.

Richardson, E., Pearce, J., Tunstall, H., Mitchell, R. y Shortt, N. (2013). Particulate air pollution and health inequalities: a Europe-wide ecological analysis. International Journal of Health Geography, 12:34.

Rollston, R., \& Galea, S. (2020). COVID-19 and the Social Determinants of Health. American Journal of Health Promotion, Vol.34, 6, pp. 687-689.

Rozenfeld, Y., Beam, J., Maier, H., Haggerson, W., Boudreau, K., Carlson, J., \& Medows, R. (2020). A model of disparities: risk factors associated with COVID-19 infection. International journal for equity in health, 19(1), 1-10.

Santana M., Rosales E., y Giovanna S. (2016). Geografía de la salud: antecedentes, aspectos teóricos y perspectivas. En Olivera José y Souza Geraldo (comp). Geografía da Saúde. Ambientes e sujeitos sociais no mundo globalizado. Ed. Universidad Federal del Amazonas. Manaus: Brasil, ISBN: 978-85-7401-817-0, pág. 95-128.

Smith. S, y Easterlow, D. (2005). The strange geography of health inequalities. Transactions of the Institute of British Geographers, Vol. 30, pp 173 - pp 190.

Sommer, I., Griebler, U., Mahlknecht, P. et al. (2015). Socioeconomic inequalities in noncommunicable diseases and their risk factors: an overview of systematic reviews. BMC Public Health 15, 914.

Stringhini, S, Carmeli, C, Jokela, M, et al. (2017). Socioeconomic status and the $25 \times 25$ risk factors as determinants of premature mortality: a multicohort study and meta-analysis of 1.7 million men and women. Lancet, 389(10075):1229-1237.

Vincens, N., Emmelin, M., \& Stafström, M. (2018). Social capital, income inequality and the social gradient in self-rated health in Latin America: A fixed effects analysis. Social science E medicine, 196, 115-122. 
